\title{
MECHANICAL PROPERTIES OF RECYCLED PAPER AND CARDBOARD
}

\author{
DRAGAN OBRADOVIC ${ }^{1}$, LAKSHMI NARAYAN MISHRA $^{2 *}$
}

1 Elementary school "Jovan Cvijic", Kostolac-Pozarevac, Mathematics, Serbia, dragishaobradovic@yahoo.com

ORCID: https://orcid.org/0000-0001-5871-6958

${ }^{2}$ Department of Mathematics, School of Advanced Sciences, VIT University, Vellore 632 014, Tamil Nadu, India, lakshminarayanmishra04@gmail.com

ORCID: http://orcid.org/0000-0001-7774-7290

\section{A R T I C L E I N F O}

Article history:

Received 2020-06-21

Accepted 2020-09-04

Available online 2020-09-04

\section{keywords}

chemicals, waste paper and

cardboard, environmental

protection, recycling processes.

\section{A B S T R A C T}

In flotation deinking, the released ink should also be detached from the substrate and dispersed in order to avoid redeposition. However, in flotation deinking the probability of removal of an ink containing particle is driven by a combination of size and surface energy. In flotation deinking a certain amount of agglomeration is preferable as the removal in flotation deinking is dependent on the size of the particle. The optimum size range is often stated to be between 50 and 150 micrometers. Size alone however is not additionally the agglomerates should demonstrate surface energy properties that are more hydrophobic than wash deinking in order to allow for stable attachment and transport by air bubbles. The presence of surfactant in the formulation has a higher value for the result. 


\section{INTRODUCTION}

Paper recycling processes can use pulp produced either chemically or mechanically. By mixing it with water and applying mechanical work, the hydrogen bonds in the paper can be decomposed and the fibers separated again. Most of the recycled paper also contains new fibers added to maintain quality. Generally speaking, deinked pulp is of the same or lower quality than the collected paper since it was formed.

Recently due to increasing chemical, energy, and pulp prices mills are exploring new ways of saving costs using new concepts for recycled processing. Some chemical suppliers offer neutral deinking where chemical costs in the pulper can be saved in addition to other process and chemical benefits Due to the complexity of the entire recycling process and the inter-dependency of each process step, it is not sufficient to only look at the pulper or flotation stage but rather the entire process.

\section{PROCESSING OF OLD PAPER}

Recycled paper can be said that almost all paper production, produced before the invention, is in the process of making wood pulp, based on the recycling of various fibrous materials (canvas, fishing nets ...). Today, recycled paper or fibrous materials are made almost entirely from waste paper. About $80 \%$ of the collected waste paper can be used for the recycling process, and about $20 \%$ of various waste. Everything that cannot be used as a fibrous material must be solved from the collected paper. These are mainly wires, staples, plasticized papers and half-cardboards, papers with UV varnish and others. Fibers obtained only from old paper can be recycled several times before they become too short and brittle, and only one of them could give the recycled paper certain properties (Jiang and Ma, 2000).

The alkalinity present in traditional alkaline porridge can cause unwanted jaundice. In order to offset such yellowing and associated brightness losses hydrogen peroxide $\left(\mathrm{H}_{2} \mathrm{O}_{2}\right)$ is often added to the pulper. In some systems, one or more substances are added to improve the bleaching efficiency of the peroxide by rendering the harmful metal ions inactive in degrading the unwanted peroxide. Finally, sodium silicate (or water-glass) is commonly used. The role of sodium silicate is complex and includes complexation of some detrimental metals and contribution to $\mathrm{pH}$ buffering in some circumstances. Water glass also helps to release ink so that it does not settle on the surface again and contributes to the agglomeration of ink particles and flotation efficiency. Finally, in many situations water-glass tends to depress the removal of filler during flotation.

\subsection{The process of making recycled paper}

Waste paper recycling includes procedures for initiating the collection and sorting of waste paper or separate undesirable ingredients. Follow the transport to the paper mill and the recycling process (Fuushern, 1993). The recycling process begins: disintegration of paper in water, followed by coarse sieving of fibrous materials. One of the most important parts of the process is the removal of printed inks on paper or flotation deinking. After removing the paint, followed by cleaning, fine sifting, washing and possible thickening and preservation of the fibrous mass. The future properties of recycled paper depend on almost every part of the processing. Therefore, during the entire procedure of control and monitoring of the properties of the samples of fibrous material, satisfactory quality was obtained, which is a condition for paper production.

\subsection{Sorting paper and cardboard}

In the first phase of waste paper collection, sorting by type of waste paper has already been performed (newspapers and magazines, corrugated cardboard boxes and other paper and cardboard packaging, books ...). But this initial sorting is relatively rough, and the input of raw materials into the paper mill is examined and classified according to their origin and processing. Collected paper often contains a variety of useless papers and a variety of hard items. Since they could cause damage to machines in the processing of waste paper, this must be done by further inspection and sorting of the paper and handed over on the conveyor belt. In addition to printing inks, paper recycling often contains various adhesives. It is least desirable and most difficult to remove so much glue that is present.

\subsection{The disintegration of the old paper}

The purpose of cellulose is to separate the fibers from the intertwined structure of the paper into individual fibers. Ink is expected to separate from the fibers in the form of fine particles. Disintegration of old paper is performed in pulper (Gvozdenović et al., 1991). As it leads along the rotation of the propeller, fibrous fibers are added to the paper chemicals, and the process is controlled with respect to pulp consistency, $\mathrm{pH}$ value, temperature and fiber time. Waste paper as a raw material enters the processing process, except for printing inks and other non-fibrous contaminations, forming sticky particles. The least favorable and least desirable are stickers, envelopes, stamps or soft binding. Glue particles originate from refractory adhesives, adhesives, coating binders, thermoplastic resins, UV varnishes and waxes. It is important to note that the most commonly used water is from the factory circular flow system.

\subsection{Streaked particle removal}

Screening serves to remove all these particles, ie. nodes that are larger than the fibers in the suspension. These can be unstretched pieces of paper, present due to insufficient moisture, etc. (Case History, 2012). Moreover, the particles can be flat pieces of plastic wrap or bags, various adhesives and sticky surfaces. If, on the other hand, they break down to create fiber size, it can lag in the pulp and later in the recycled paper.

The sieve is made by passing the pulp through a sieve with a hole of a defined diameter. Sieves may have holes or slits, the sieving system often works under the pressure by which the fibers are pushed through the sieve. 


\subsection{Deinking flotation}

Deinking flotation is a selective separation process that uses air bubbles to remove paint particles from a stretched suspension. In the flotation cell, color particles are accepted on air bubbles that carry them to the surface (Križan, 1997).

Flotation chemicals are added to the pulp to increase the hydrophobicity of the paint particles and increase the flotation efficiency. Foam is created on the surface which must be removed. The efficiency of flotation depends on the probability that three conditions are met for each particle color, such as: collision particles and bubbles, acceptance of bladder particles, and final removal of bubbles with color particles from the pulp.

Many parameters affect flotation efficiency and can be classified into four groups:

1. The first properties of particles, such as size, number, shape, surface chemistry, etc.

2. Properties of bubbles, also their size, number, surface chemistry and dispersion, flotation,

3. The third degree of interference, ie. time and intensity

4. Diameter. process conditions, e.g. types of paper waste in the process, amount of ash, fiber properties, $\mathrm{pH}$ of the suspension, temperature and so on.

Lighting slurry is often a measure of the efficiency of flotation paint removal by removing ink from the system, which increases the brightness. Flotation efficiency is determined by image analysis. This method can determine the number and size of particles present in the color, size and area they occupy on the laboratory list after flotation. The assessment of flotation efficiency refers to the maximum reduction of fiber losses during the recycling process.

\subsection{Rinse and thickening}

Rinsing is a mechanical process by which the pulp is separated from the diluted dye, filler particles and other impurities. The rinsing process is dirt particles. Most of the water that contains the washed paint is drained from the pulp through sieves or passing through small particles of paint and dirt, but without fibers. Fat concentration of thickening It follows from 0.6$0.7 \%$ of the concentration of about $8-10 \%$ of dry matter. A further procedure is a mass dispersant. It is time for the glue to disperse and the ink residue to disintegrate into very fine particles, which are harmless to paper. The mass then went to the pool, where it was used on a paper machine.

\subsection{Changing the properties of recycled paper}

Papers made from recycled fibers differ from virgin paper in many respects. By rough division, these differences can be classified into optical and physical properties (Križan, 1997).

The cause of these differences are primarily changes in the fiber itself, and then the presence of various impurities that were sufficiently removed from the pulp during recycling. Efforts to effectively remove impurities in the form of particles can be manifested in negative weight loss, ie. which contains the use of the whole process, making it less economical. Since the screening and cleaning of the pulp during recycling can only remove part of the impurities, the remains of impurities will remain in the suspension of secondary fibers. If there is a lot of sticky particles in the dirt, the layers of paper wound on the roll can be bonded together to stick, which will cause the paper strips to tear during printing. Adhesive particles often make monitoring and recycling difficult. They usually come from glue that enters the system with raw materials, waste paper. Adhesive particles are often the cause of holes and cracks in the paper on the way to sticking to the screen of the paper machine, so the paper tape moves to other stages of the paper machine, pressing, drying, processing in places where sticky particles are missing cracks in the paper tray. So, this type of contamination makes the operation of a paper machine that is often stopped, cleaned and inspected.

The presence of contamination in the paper produced from secondary fibers can also affect the strength of the paper, although in this area the effect of the changed properties of the fibers themselves is much more significant. Pollution particles can affect their presence on the connective fibers. The fibers near such particles cannot be well interconnected, so there is a crack in the list of structures. When you insert paper into the press, especially the role of the press, this defect can affect the tearing of the entire strip of paper.

\subsection{Advantages of recycled paper}

Processing of waste paper used for writing and for all cardboard packaging. Magazines can be recycled at least 7 times. Cardboard packaging is usually made from recycled paper as well as from newspapers. Paper recycling saves them in some way.

The level of paper recycling in the world: Japan 48.2 percent, USA 27 percent, Sweden 55 percent, 60 percent of the Netherlands, Denmark 30 percent. The factories produced original ecological paper, recycled paper and cardboard for the environment (Case History, 2012). Original paper without bleaching and dyeing with minimal energy and water consumption produced from $100 \%$ waste paper. Recycled paper is produced from $80-100 \%$ cellulose waste paper with the addition of new chemical auxiliary raw material. Cardboard in real average $90 \%$ waste paper. Recycled paper is used for copiers, opaque is 4-5 times less harmful to the environment, and its life is over 100 years. It is used in the paper industry, construction (as an insulating material) and the furniture industry (plywood replacement).

\section{PROCESS PAPER RECYCLING}

Paper recycling is done by cutting paper soaked in water, grinding it into a pulp, dye can be added, pressed and then dried. Paper can be recycled only 5 to 7 times before it disintegrates: the fibers in it burst and break into a stable network that makes paper (Gvozdenovic et al., 1991). Therefore, you must add fresh, new paper fibers to each sheet of recycled paper. Energy-saving paper protects forests, but also the energy needed to prepare it. Cardboard packaging for milk and juices differs from ordinary cardboard and paper, according to the method of use and the method of collection, sorting and recycling. There are two types of packaging for milk and juices. Aseptic packaging is used for packaging UHT milk and juice, so that packaged products do not have to stand in the refrigerator before opening and use. Another type of cardboard packaging used for switching between short-term pasteurized milk and dairy products and should be refrigerated before using the packaged product (Merriman, 1993). 
Cardboard packaging has one or more crossed plates, which gives strength, then $20-25 \%$ of plastic called polyethylene and a packaged product that protects against moisture and bacteria. There are many of them in aseptic packages of a thin layer of aluminum foil which prevents oxygen and light rays from spoiling the packaged product. Packaging waste should be recycled into new soil materials, in order to reuse the raw materials from which the packaging is made. Cardboard packaging for milk and juices can be collected together with waste plastic and aluminum packaging or together with cardboard and paper. Whichever way it is collected, this packaging is recycled in a paper factory after separation from other types of waste.

\section{RECYCLED FIBER (pulp recicled)}

The reuse of waste paper for the production of cardboard and paper is today economically and environmentally justified, because it saves large amounts of wood (Nie and Miller, 1997). There are fairly clean (and cut leaves from the coil) to heavily contaminated with 3\% impurities. Many types of waste paper are used in the recycling and separation process. About $80 \%$ of the collected waste paper can be used for recycling, and about $20 \%$ of the waste: wire, staples, plastic materials and adhesives that are the most difficult to remove. Paints and varnishes must be removed during fibering, cleaning and sieving. Recycled paper begins to disintegrate in water, followed by coarse sifted fibrous material. One of the most important parts of the process is the removal of printing inks on paper, flotation deinking (Fig. 1). After removing the paint, it is followed by cleaning, fine sifting, washing and eventual thickening and preservation of the fibrous mass. The future properties of recycled paper depend on almost every part of the processing. Therefore, during the entire process, the samples are controlled and the properties of the fibrous materials are monitored in order to obtain a satisfactory quality, which is a prerequisite for the production of paper and cardboard. (Fig. 1).
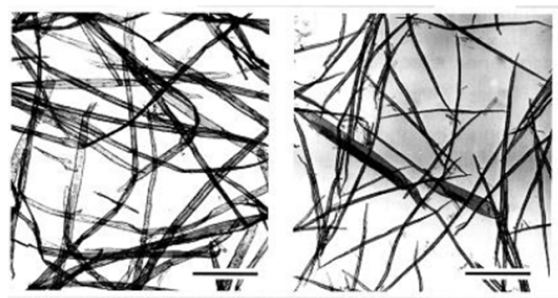

Fig. 1. Conifers and deciduous Source: (Krgović, 2005)

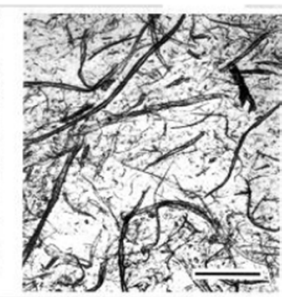
wood pulp

4.1. The strength of a sheet of paper

Strength testing of fiber extracted from the leaves of paper that have been subjected to frequent recycling determine that such individual fibers of each subsequent cycle slightly less stretched and at the same time becoming firmer, stronger. These tests confirmed the crucial importance of connections fiber - fiber to the paper. The strength of the fibers is insufficient if the volume of paper fibers in the sheet is not sufficiently linked to each other and intertwined. Recycled paper sheets in frequent recycling all show less resistance to cracking, tearing, stretching and pulling out the lower porosity and density of the list.

\subsection{Changes to the paper fibers due to the recycling}

Of paper from recycled fibers differ from paper from virgin fiber among other things, for having weaker mechanical properties.

To study the volume of paper from recycled fibers important properties of the fibers themselves, as follows:

A - speed fiber,

B - length of fibers,

C - swelling of the fibers or their plasticity,

D - potential bonding fibers.

Ad A) The strength of the fibers is usually measured as the prime range breaking length of paper.

Ad B) length of the fibers plays an important role for the strength of the paper. The process recyclable fibers become rigid, and therefore brittle and break in REFINER, although it is not implemented lean grinding (Peretin, 2010).

Ad C and D) The connection of fiber - fiber affect two parameters: the first, which can be attributed to the swelling fibers or its plasticity, determines how the fibers adhere to each other, therefore refers to the surface of the fibers which is bonded with other fibers. It is known that the recycled fiber is less pliable than the primary fibers. The second parameter is the specific bonding strength. The mechanism of binding fiber - fiber includes the impact of hydrogen bonds, while the fiber after drying potential of hydrogen bonds can be reduced. The observation of these four parameters is crucial for studying the volume of paper from recycled fibers. Since the combination of paper is incorrectly bonded cellulose fibers, its strength is also the bond strength of these fibers.

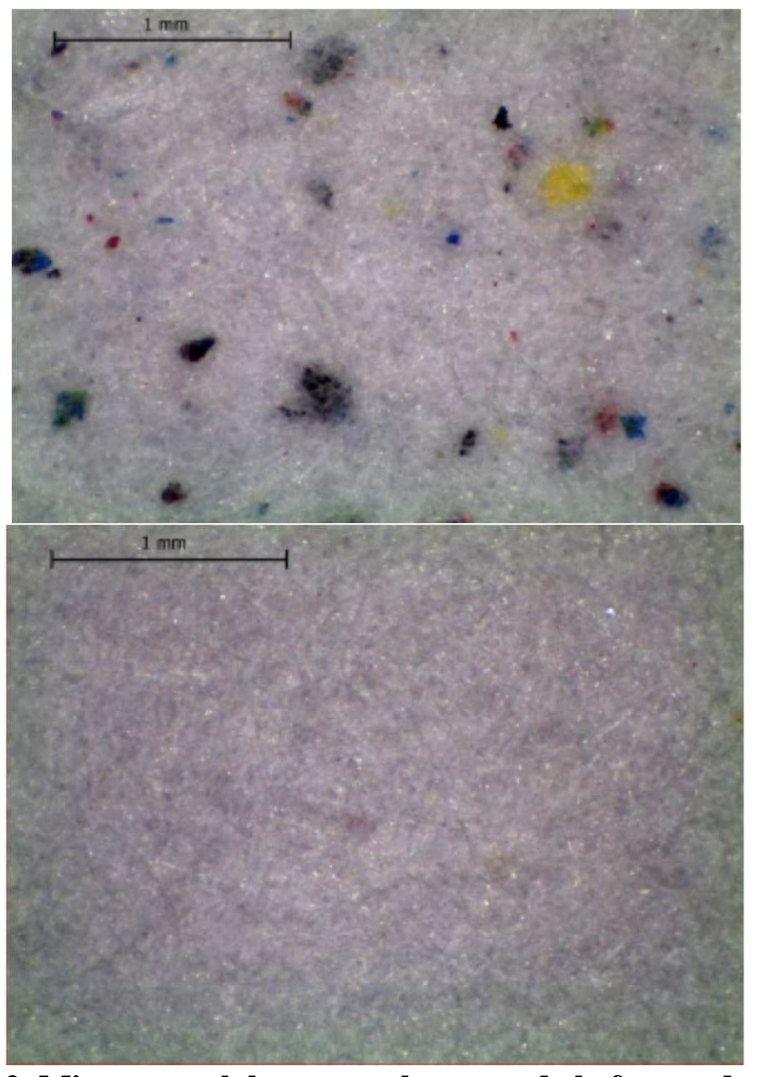

Fig. 2. Microscopy laboratory sheets made before and after flotation

Source: (Trumic, 2015) 


\section{FROTH FLOTATION}

Froth flotation is a process for selectively separating hydrophobic materials from hydrophilic. This is used in the mineral processing, paper recycling and wastewater treatment industries. Foam flotation cell diagram.

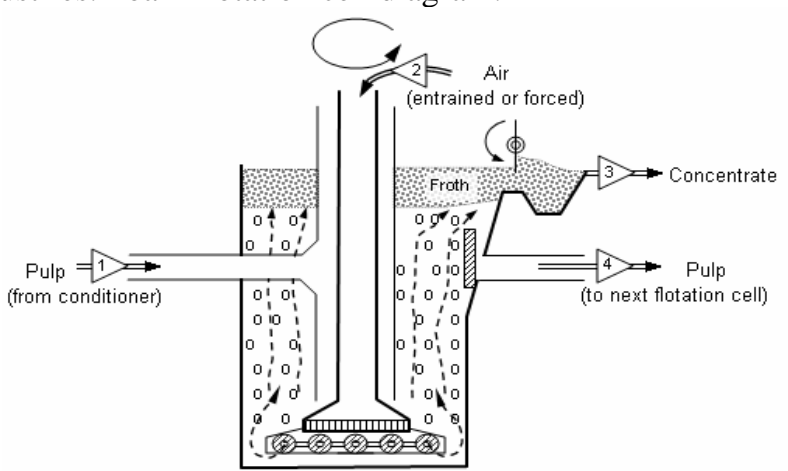

Fig. 3. Diagram froth flotation cell

Source: https://en.wikipedia.org/wiki/Froth_flotation

\section{CHEMICAL DEINKING FLOTATION}

Flotation is a complex chemical process in which hydrophobic suspended, colloidal and partially dissolved particles are removed with the help of gas bubbles. Foam (separated impurities) is formed on the surface of the liquid, which is constantly removed by skimmers (paper recycling, 2016). In flotation, there is an extremely complex liquid-gas system, so that a large number of complex phenomena occur. Since the particles present in the suspension are often hydrophilic, in order for the flotation process to be efficient, it is necessary to change the moisture properties of the particles present with the help of a surfactant with polar-nonpolar molecules. The molecules are adsorbed on the surface of the hydrophilic particles by the orientation of the hydrocarbon part of the molecules according to the volume of the liquid, thus making the complexes hydrophobic.

Flotation takes place mainly in alkaline media ( $\mathrm{pH} 7-10)$, with a solids content of $0.7-1.5 \%$, and the chemicals used in the flotation process are fats and mixtures of products in the processing of oil, wood and coal, hydrocarbons acids (C4 C20) or organic compounds with sulfate ion. When processing waste printed paper, it can be noticed that the flotation process takes place through two phases as primary flotation and secondary flotation.

In primary flotation, 6 to 10 cells are required in a row to efficiently separate graphic inks and other impurities from the system, while in secondary flotation, usually through 2 flotation cells, foam (floating impurities) is purified to reduce fiber loss. Between the two phases of flotation, there is an intermediate phase of dispersion (solids content 22-32\%), which has the role of additional disintegration of graphic colors and bleaching of fibers in order to achieve the appropriate whiteness. Sodium hypochlorite $(\mathrm{NaOCl})$ and hydrogen peroxide $\left(\mathrm{H}_{2} \mathrm{O}_{2}\right)$ are most commonly used as optical brighteners for cellulose fibers. The use of silicates and chelates is also necessary to deactivate metal ions (present in the waste paper), which could catalytically decompose peroxides. If recycled cellulose fibers are mixed with fibers obtained by wood processing after refining (combined pulp production process), the bleached cellulose phase is not required.

\subsection{Industrial deinking procedure}

The most commonly used industrial deinking method is called froth flotation. The principle behind this process is the separation of hydrophobic and hydrophilic particles in the pulp by a stream of air bubbles, after mechanical and chemical treatment of the paper pulp. In order to implement proper flotation-based deinking, a flotation cell is needed (Fig. 4).

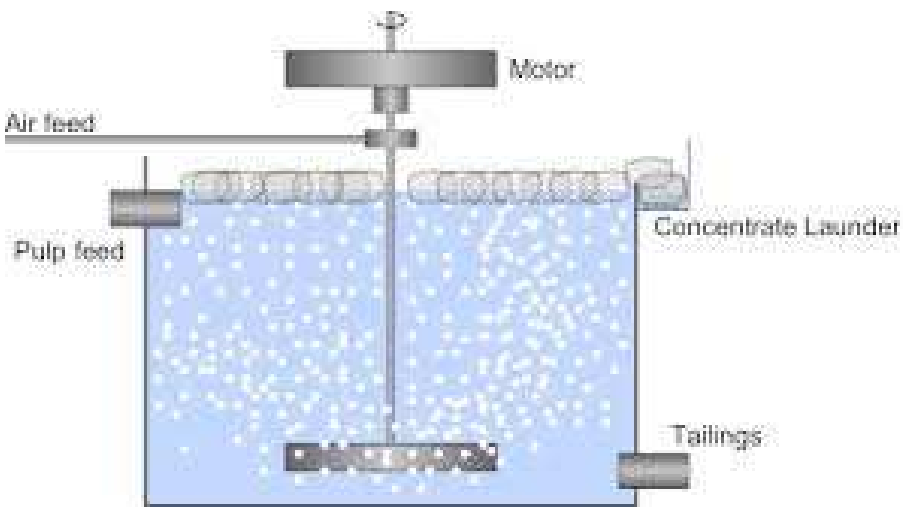

Fig. 4. Flotation cell

Source: https://www.google.com/search?q=Deinking+flotation

\subsection{Deinking chemistry}

The goal of the deinking process (Lassus 2000) is to produce insulated pulp from recovered paper (RCP) with sufficient lightness and purity to meet the quality requirements set by the quality of the paper produced. A prerequisite for this action is that the fiber-bound ink content, dirt content and ink fragmentation are minimized in the cellulose phase. It is therefore important to identify the factors influencing the ink behavior in cellulose, to look for new ways to improve the separation of ink from RCP into pulps, and to search for cellulose methods that efficiently process RCPs printed with various inks. Fig. 5. Schematic representation of the paint removal plant. In traditional alkaline paint removal, these devices often consist of fatty acids and / or nonionic surfactants.

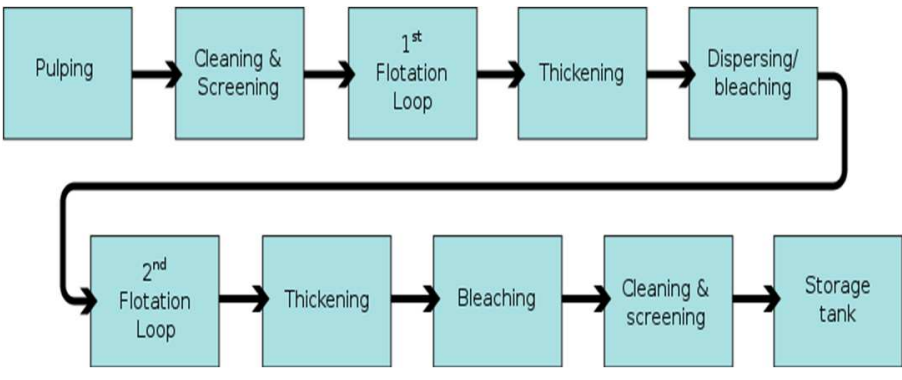

Fig. 5. Schematic layout of a deinking plant

Source: https://www.google.com/search? $q=$ Deinking +flotation

In systems that use fatty acids containing deinking aid level of water hardness can be important. Often a source of calcium such as calcium chloride is added to the process to ensure that the soap fatty acid is converted into an insoluble calcium salt, which is believed to be desirable fatty acids speciation ink collection in flotation. 


\section{REMOVAL OF INK FIBERS DURING REPULPATION}

Changes in the printing properties of recycled paper relative to primary fibrous paper are closely related to physical changes on paper. The physical properties of the paper dictate the continuous printing process and apply to all situations where printing may be slowed or interrupted, either due to tearing of the paper strip, or tearing or dusting of the sheet surface, or insufficient dimensional stability of the paper.

Flotation deinking is one of the steps of deinking, which usually consists of removing ink from the fibers during repulpation, followed by flotation and washing the separation of ink from the suspension. Today, paint removal is done by the flotation method. Flotation is a separation method that uses different surface stone properties of different particles. The slurries that come from the pre-creation with a consistency of $2-3 \%$ are diluted to a flotation concentration of $1-1.4 \%$.

Patented flotation for energy-saving paint removal:

$\checkmark \quad$ Self-cleaning system, without plugs and without wear with low service requirements,

$\checkmark$ Automated controlled flow foam,

$\checkmark$ Indoor aeration unit resulting in a narrow circuit of process air,

$\checkmark$ Smart modular construction Your advantages,

$\checkmark$ Best possible brightness enhancement and maximum dirt stain removal for particle sizes between 5,500 $\mu \mathrm{m}$.

Factors to consider when choosing a paint remover, choosing the right paint remover is based on a variety of factors. In each series in increments of $20 \%$ decreasing share of conventional offset prints on newspaper and at the same time increasing the share of digitalog offset print. In two meals per $44 \mathrm{~g}$ sample is added to 2 liters of tap water temperature $50^{\circ}$ $\mathrm{C}$, and flotation agents in the order shown in table 1 (Cuts to $88 \mathrm{~g}$ sample parts format about $4 \mathrm{~cm}^{2}$ ).

\begin{tabular}{|c|c|}
\hline Ordering & Type of flotation agents \\
\hline 1. & $0.3 \mathrm{ml} 50 \%$ Flokutana \\
\hline 2. & $1.54 \mathrm{ml} 10 \% \mathrm{H}_{2} \mathrm{O}_{2}$ \\
\hline 3. & $1.73 \mathrm{ml} 5 \% \mathrm{NaOH}$ \\
\hline 4. & $0.053 \mathrm{ml} \mathrm{Aquasec}$ \\
\hline
\end{tabular}

Table 1. Ordering and kind of flotation agents for laboratory recycling

In a desintegrator to the disintegration of samples is performed for 40 minutes, after which a disintegrated sample is transferred to the mixing vessel where it was diluted with an additional $4 \mathrm{~L}$ of water temperature $30^{\circ} \mathrm{C}$.

\section{CONCLUSION}

The present level of development of technology for recycling waste materials, particularly waste paper and cardboard, has reached such a value that can meet environmental criteria and economic parameters, which is certainly the focus of investment and accelerated construction of processing facilities. The mere fact that in Europe concentrated research and development of technical and technological knowledge and experience and that is a big exporter collected and recycled paper and cardboard.

The quality of newsprint as a representative of the printing recycled paper, qualifies the optical and mechanical properties and surface characteristics. On these properties are in the process of recycling can influence choice of paper and printing techniques as prints and procedure of recycling, which was the goal of this research.

The research results indicate that the mechanical properties of recycled sheet depend on the complex influence of several factors. They include not only the properties of the printing substrate and the mechanism and conditions recycling process, but also press the principles of individual printing techniques.

\section{REFERENCES}

CASE HISTORY. The Truth about recycling. The Economist, 2012.

FUUSHERN, W. Mechanical properties and structural changes in recycled paper. Ph.D.Thesis, University of New York, 1993.

GVOZDENOVIC, J.; CURAKOVIĆ, M.; LAZIC V. Recycling of packaging materials in the process industry and its ecological significance. Environmental Protection, Proceedings, v. 194, Dubrovnik, 1991.

JIANG, C.; MA, J. Deinking of waste paper: flotation. Encyclopedia of Separation Science, Academic press, p. 2537-2544, 2000.

KRIŽAN, M. Contemporary paper production. Belgrade: Mrlješ, 1997.

LASSUS, A. Deinking chemistry. In: Göttsching L \& Pakarinen H (eds) Recycled fiber and deinking. Helsinki, Fapet Oy: 240-265, 2000.

MERRIMAN, K. Cleaning for contaminant removal in recycling - fiber systems, in A secondary fiber recycling. Ed. Spanenberg, R.J., TAPPI Press, Atlanta, p. 101- 122, 1993.

NIE, X.; MILLER, J.D. The Effect of Ink Types and Printing Process on Flotation Deinking. TAPPI Proceedings Recycling Symposium, p. 131-147, 1997.

PAPER RECYCLING. Recikliraj.hr, available at: http://recikliraj.hr/recikliranjepapira/, accessed: 19 March 2020.

PERETIN, S. Improvement of household waste sorting. Final thesis, University of Zagreb, Faculty of Mechanical Engineering and Naval Architecture, Zagreb, 2010. 